\title{
CONGENITAL DEFECTS IN THE PELVIC PARASYMPATHETIC SYSTEM*
}

\author{
BY \\ ORVAR SWENSON \\ From the Department of Surgery of the Boston Floating Hospital and Tufts College Medical School
}

Nine years ago, while observing a group of patients with congenital megacolon, three children came under my observation who had transverse colostomy. This vent in the colon had relieved the patients' symptoms completely. The colostomy was their only remaining problem. After discussing the patients with my colleagues, it was decided that the colostomies should be closed and medical therapy instituted. This programme was followed, and within seven months all of the patients' symptoms recurred despite careful medical management. Re-opening the colostomies again relieved all of their symptoms.

In reviewing this experience, it seemed obvious that such patients must have some form of functional obstruction in the colon beyond the colostomy. Careful barium enemas were performed on other patients with congenital megacolon, and in the distal portion of the large intestine there was a persistent narrowing of the lumen beyond the dilated colon which varied in length from patient to patient, but otherwise was quite similar. Usually the rectum and rectosigmoid and varying parts of the sigmoid colon were irregular and narrow in calibre, and the colon above this narrow portion was considerably dilated (Fig. 1).

In the course of performing transverse colostomies on patients with Hirschsprung's disease, explorations were performed and the lesion in the distal colon was examined. The dilated left colon was markedly hypertrophied and within a distance of 5 to $8 \mathrm{~cm}$. the calibre of the bowel abruptly narrowed so that the lumen became smaller in diameter than normal. The narrow segment was little if at all hypertrophied.

A group of patients with transverse colostomies was accumulated, and these afforded an opportunity to study the motility of the left colon. A multiple balloon technique was used in which one balloon was located in the splenic flexure, one in the

* The 1954 Alex Simpson-Smith Memorial Lecture delivered at the inaugural meeting of the British Association of Paediatric Surgeons at The Hospital for Sick Children, London. descending colon, and one in the narrow rectosigmoid. A number of normal individuals were studied, and it was found that in the normal intact left colon peristaltic waves progressed in an orderly fashion from the splenic flexure through the rectum. Patients with megacolon exhibited marked variations from the normal. The peristaltic waves progressed from the splenic flexure to the descending colon in an orderly manner. However, the balloon in the rectosigmoid failed to record any progression of the peristaltic waves into the narrow portion of the colon. There were frequent, feeble, purposeless contractions which were unrelated completely to the normal peristaltic waves in the dilated colon above (Swenson, Rheinlander and Diamond, 1949). These studies led to the conclusion that there was a segment

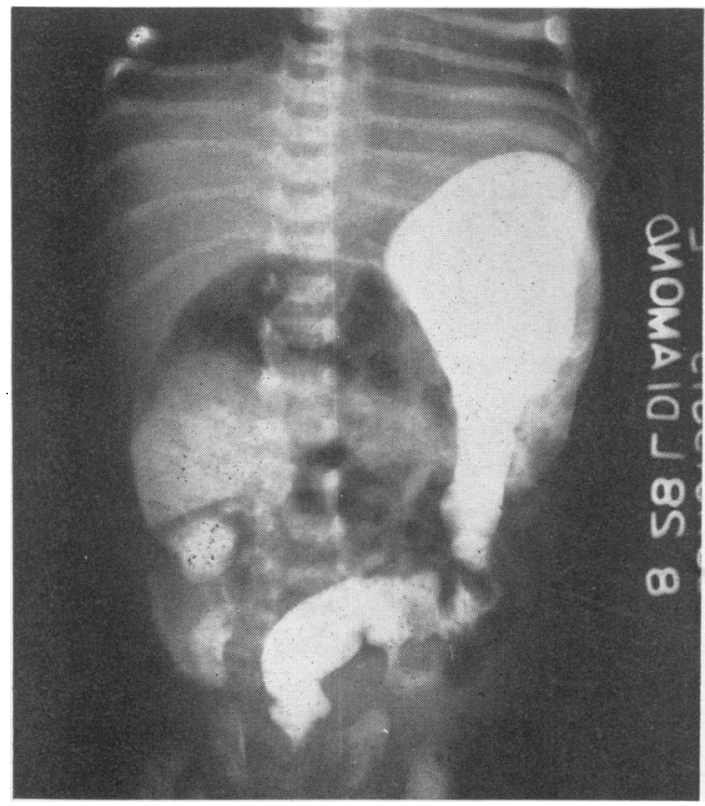

Fig. 1.-Appearance of a barium enema in a patient with Hirschsprung's disease. This lesion was higher than usual, being in the splenic flexure. 
of colon in these patients which was without peristaltic activity. It was tempting to postulate the presence of spasm in the colon beyond the dilatation, and there was some evidence that this was true. However, it was not possible to prove this point definitely by direct measurements.

It was concluded from these studies, as well as from our observation of the patients with colostomy, that if the inactive segment of bowel were resected and continuity of the intestinal tract were restored, these patients should be relieved of their functional obstruction. In the laboratory a technique was tested which consisted of resection of the rectosigmoid and rectum with an anastomosis between the anal canal $1 \frac{1}{2} \mathrm{~cm}$. from the mucocutaneous margin and the colon above. Dogs were used in these studies, and it was found that the technical procedure was feasible and that the animals were continent post-operatively. This technique was utilized on patients and the results were most gratifying (Swenson and Bill, 1948).

Clinicians were slow to realize the significance of the absence of ganglion cells which had been described by a number of workers (Dalla Valle, 1920; Robertson and Kernohan, 1938; Tiffin, Chandler and Faber, 1940; Zuelzer and Wilson, 1948). In this series of 150 cases absence of ganglion cells has been a constant finding. The absence of ganglion cells extended to the internal sphincter in all except one of the specimens removed at operation in this series. This patient had a cuff of distal rectum 4-6 cm. long with normal ganglion cells and proximal to this a segment in which the ganglion cells were absent. Our experience has been that there is a total absence of ganglion cells except in the transition zone adjacent to the normal colon. Here, in a band $2-3 \mathrm{~cm}$. in length, there is a decrease in concentration of ganglion cells.

In a review of this group of 150 patients it was found that the most important point in the history was the duration of the disease. Virtually all of the records revealed a history of symptoms dating from birth. This is in contrast to the children with habit constipation where the onset of symptoms is usually at 2 or 3 years of age. On examination of these patients, the finding of an impaction in the sigmoid and no impaction in the rectum is strong evidence of Hirschsprung's disease. Barium enema must be used to confirm the diagnosis of Hirschsprung's disease, and in the majority of patients the diagnosis can be made unequivocally.

In the past few years I have become increasingly aware of the difficultty in diagnosing Hirschsprung's disease accurately in newborn babies and infants under 6 to 8 months of age, for in this age group the barium enema often fails in making a definite diagnosis. Since the aganglionic segment in most patients extends down to the internal sphincter, we determined to biopsy the rectal wall through the anal canal in an attempt to develop a satisfactory biopsy technique for positive diagnosis of Hirschsprung's disease. Such a biopsy would also be useful in older patients who had equivocal signs of the disease on radiological examination. A third group of patients presented themselves who posed a diagnostic problem. These were the children who had had a transverse colostomy early in life. Whether or not these children had Hirschsprung's disease was important in their management. Placing barium in the deactivated colon distal to the colostomy was of no avail because this segment had become uniformly small in calibre. In order to test the validity of our biopsy technique, we performed the procedure on 10 cadavers. We learned that the internal sphincter itself was hardly a reliable source of biopsy material, but that immediately above this in the rectal wall a sufficient amount of rectal wall could be removed to make the diagnosis.

In this series of 10 cadavers we removed a triangular piece of rectal wall about $1 \mathrm{~cm}$. in length and $\frac{1}{2} \mathrm{~cm}$. in width. All of these proved to have ganglion cells. We have subsequently biopsied a group of 19 patients who at operation proved to have Hirschsprung's disease. In all of these no ganglion cells could be found in the biopsy material: This test has proved to be of considerable value in the three groups of patients which $I$ have enumerated. An adequate sample of the rectal wall must be removed. We have now performed the biopsy on a total of 40 patients, and in none has there been any complication except bleeding in one patient postoperatively. Of the total number, 19 were aganglionic and proved to have Hirschsprung's disease; 21 had ganglion cells in the biopsy and these patients had histories and radiological findings consistent with chronic constipation. This seems a satisfactory and helpful technique in the various diagnostic problems with which one might be confronted in considering the diagnosis of congenital megacolon.

There are several points in the operative treatment of these patients that should be stressed. In the first place we are now resecting $75 \%$ of our patients without a preliminary colostomy. The multiple stage method of management is reserved for those patients who are extremely ill and emaciated. Colostomy is most frequently used in infants, for in our experience more severely ill patients are encountered in this age group than in any other. The colostomy should be performed in the left colon at the level of the junction of the aganglionic and 
normal colon. This can be determined by performing a biopsy of the muscular coat at the proposed site of the colostomy. The bowel wall is incised down to the mucosa and the muscular coat freed for 4 to $5 \mathrm{~mm}$. and a full thickness of muscular wall excised. This is immediately studied by frozen section and if the ganglion cells are found the colostomy is performed at this site. If none are found, the colostomy is placed at a higher level where the neuromuscular structures are intact. The advantage of a left-sided colostomy at the junction of the aganglionic and normal colon is that at the second procedure the colostomy can be resected along with the aganglionic colon and continuity of the intestinal tract established. Thus the programme is reduced to two operative procedures. Formerly, when the colostomy was performed in the transverse colon, we were committed to three operations: colostomy, resection of the aganglionic segment of colon with anastomosis, and then closure of the colostomy.

Preparation of these patients for operation is less elaborate than formerly. The parents are instructed to administer daily enemas to the child for three days preceding admission to hospital. No chemotherapy is used to prepare the intestinal tract. It is possible to be less vigorous in the preparation of these patients when a segment of the colon is resected during the abdominal phase of the operation. Large faecal impactions can be removed in this manner. If one utilizes the telescoping type of procedure in which there is no resection of bowel during the abdominal phase of the operation, a more elaborate preparation of the gastro-intestinal tract must be performed. I used the telescoping operation in my first few patients and found that it was so clumsy that it was discarded for the type of procedure now used. There are additional advantages in the resection type of procedure. A biopsy is secured from the proximal end of the resected bowel, and this ensures that the whole aganglionic segment has been removed. Another often unappreciated advantage is that one has a very small length of rectum and rectosigmoid to pull down through the anal canal. This is much neater and cleaner than everting the whole dilated intestine. The greatest advantage of all is that should one pull the short segment of bowel down and find that it has not been freed low enough, the small segment can be returned to the pelvis with little difficulty and the dissection continued. If the whole segment has been telescoped, it is an arduous task to reduce the intussusception to permit a completed dissection.

The second technical feature of the operative procedure that should be stressed is that in order to ensure a good blood supply at the proximal line of resection, it is advisable to free the splenic flexure and to divide the left colic artery. A more limited resection was performed early in my experience, and at times the blood supply was precarious in the proximal segment.

A few patients have been encountered with extensive lesions, some of which have extended to the transverse colon and even to the hepatic flexure. In these patients we had divided the middle colic artery and vein and rotated the ascending colon $180^{\circ}$ down into the pelvis, and performed the reconstruction in this manner. It is surprising how well these patients have functioned post-operatively.

I have encountered two infants with the entire colon aganglionic, and the management of these babies was problematical. Initially both of these infants had ileostomies closed. An experience six years ago with a patient suggest a method of managing these two infants. This boy had a colectomy with an anastomosis between the ileum and rectosigmoid. With the short aganglionic segment in the pelvis, the proximal ileum dilated to enormous size, abdominal distension was massive, and the child was critically ill. It was not until ileostomy was performed that he began to gain and do well. Again in this boy the problem was what to do with the ileostomy. After considerable discussion with colleagues and with the parents, the rectum and rectosigmoid were resected and the terminal ileum anastomosed to the anal canal, utilizing the same technique of anastomosis as is used with the routine patients with Hirschsprung's disease. It took some time for the boy to adjust to the situation and he did have some rather hectic times. Gradually, over a period of months, he improved. He is now six years from operation and has grown and developed perfectly normally. He has no further troubles and only a moderate amount of dilatation of the terminal ileum. He has had two or three semi-formed evacuations daily; he is perfectly continent. On the basis of this experience it was decided that the same procedure should be performed on these two infants. Surprisingly their course was less complicated than the older child's had been. Immediately postoperatively they had as many as $\mathbf{1 5}$ loose stools a day, and the perineal care was a constant nursing problem for a matter of several weeks. Gradually the stools became firmer and movements were reduced to three or four a day. These two infants are now one and a half years and one year from operation, and their growth and development have been normal (Fig. 2). Barium enemas show some dilatation of the terminal ileum but far less than had been anticipated. 
The next important technical detail of the operative procedure is the anastomosis. It must be made water-tight or leakage and pelvic infection may occur. First the muscular coats of the two segments are approximated with interrupted 00000 silk. This row must be placed completely around the bowel, and all the sutures tied before the proximal segment is opened. Thus, the first layer is made under aseptic conditions and the pelvis is protected from contamination by this suture line. When it has been completed, the proximal end is opened and the mucosa of the two segments are then approximated with interrupted chromic catgut sutures.

In our series of 150 patients, we have had three patients who have had leaks at the anastomosis. One of these was a patient who had such a short segment of bowel proximally that we could not complete the anastomosis, and we realized that some pelvic infection would be inevitable. The infection was surprisingly limited; however, this child developed a severe stricture which has been the source of chronic trouble. We have had five other minor strictures, all of which have yielded to digital dilatation. The second patient with infection had a perirectal abscess which was easily drained. Fortunately a fistula did not develop. The third patient with infection developed a rectovaginal

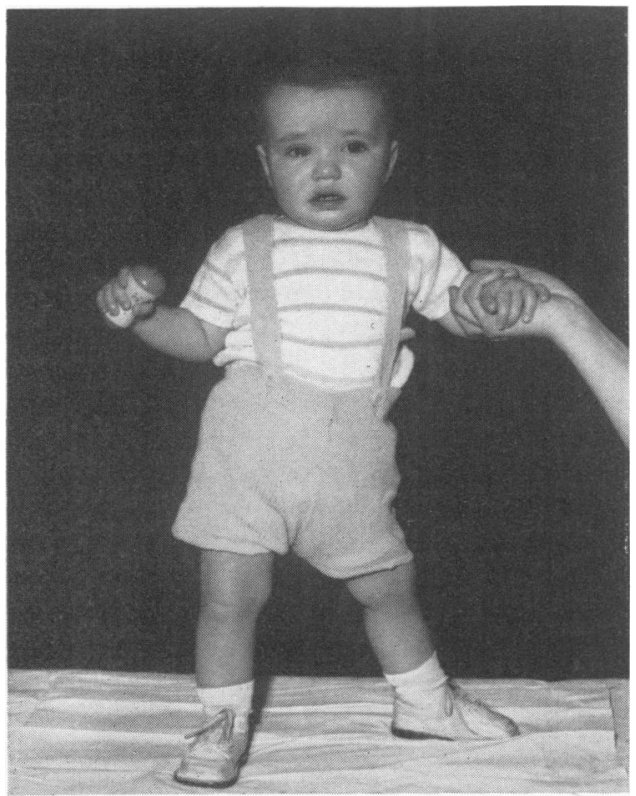

FiG. 2.-Photograph of child whose entire colon was aganglionic This was taken one and a half years after colectomy and anastomosis of the terminal ileum to the anal canal. This child is continent and growth and development have been normal. fistula. This was probably due to a suture placed too deeply so that it perforated the vaginal wall. In this particular patient a small fistula developed, and a transverse colostomy was performed and she was left with a diverted faecal stream for six months. The colostomy was closed two years ago, and the fistula has not recurred. No operative procedure was performed in the region of the fistula.

Post-operatively our patients have done uniformly well and have been relieved of their symptoms. It has not been necessary to resort to laxatives or enemas in any of our patients post-operatively, except in three children who for a short time developed mild constipation at the age of 2 or 3 years. All occurred some months after operation and all are doing well now.

In this series, 20 patients were operated upon in the first six months of life, and in this group there were four post-operative deaths. Of the four, one had a multiple stage procedure and three had primary resections. In the remaining 130 patients, there was one post-operative death. This $2 \frac{1}{2}$-year-old boy died with generalized convulsions, probably due to pulmonary infection and septicaemia. The abdomen and pelvis were free of infection. It is questionable whether the outcome would have been more favourable if the three with primary resections who died had been treated with prese liminary colostomies. Of these three, the causes of death were intestinal obstruction, recurrence of the disease because of incomplete resection and bilateral adrenal haemorrhage. The patient with the multiple stage procedure had congenital heart disease.

There is one complication to which these patients seem to be prone which is worthy of careful consideration. For some obscure reason, some of these children with Hirschsprung's disease do not tolerate infection in a normal manner. This is particularly true of the so-called gastro-enteritis which we consider to be an intestinal infection of viral aetiology. A normal child with gastro-enteritis has a short illness with vomiting and diarrhoea. Some children with megacolon either pre- or postoperatively become desperately ill under similar conditions. Initially there may be vomiting and a few diarrhoea stools. Later progressive abdominal distension and obstipation develop. Profound dehydration occurs from loss of fluid into the gastrointestinal tract as well as from the vomiting and diarrhoea. Part of the derangement of function is spasm of the sphincter mechanism so that nothing is released from the intestinal tract. This disease is most commonly seen in small infants. Treatment must be directed along two lines; first, dehydration must be combated, and as this is often greater 
than anticipated, generous parenteral replacement must be provided. Second, a large soft rectal tube must be inserted through the anal canal, and the colon drained and gently irrigated with warm normal saline. Irrigation must be repeated three times a day. Usually in three or four days the child is perfectly well, and the stools become normal in consistency, and the children return to their usual habit of one or two evacuations daily. Most children with Hirschsprung's disease never have these attacks. Some seem to be more prone to them and to have them repeatedly. However, the tendency is that the attacks are less severe and less frequent as the child grows older.

Faecal incontinence has not occurred in our patients except for some soiling immediately postoperatively. All have been continent at the time of discharge. There has been considerable discussion about the problem of impotency following resection of the entire aganglionic segment. Some time ago State (1952) suggested anterior resection as the treatment of choice on the assumption that a more extensive operation would result in impotency. We have now operated on six adult males and in each instance ejaculation is normal post-operatively. In view of this, it seems illogical to perform an incomplete resection of the aganglionic segment on the supposition that this will result in impotency. It is very important in doing the resection that the rectum be freed by dissecting right on the bowel wall so that there will be no disturbance of the automatic fibres in the pelvis. This has another useful purpose, for a number of these patients have some defect in bladder function and unless the operation is performed with the greatest care there may be damage enough to produce further atonia of the urinary bladder. In our group of 150 patients there has been one who had some difficulty emptying the bladder post-operatively. She was placed on catheter drainage with a tidal irrigator for two weeks, and during this period bladder tone returned, and her urinary function has remained satisfactory. This complication came early in our experience, and since that time an indwelling catheter is routinely left in place for eight to nine days post-operatively. Stephens, who worked with me in Boston, returned to England, and, using our technique, reported good clinical results (Bodian, Stephens and Ward, 1950). Hiatt, using the same operative technique, reports a large series of good results (Hiatt, 1951).

Early in our experience it became evident that some of our patients with Hirschsprung's disease had urinary bladder dysfunction. This led to studies of bladder function pre-operatively in all of our patients of sufficient age for the performance of satisfactory cystometrograms. A considerable number had some deviation from normal in the cystometrograms. These changes consisted of greater capacity and less vigorous detrusor contractions than normal. In $5 \%$ there was considerable derangement of bladder function and dilatation of the upper urinary tract. These patients with megalo-ureters were carefully studied to be sure that they had no mechanical obstruction at the bladder outlet or at the uretero-vesical junction. I began to consider the possible anatomical defects which might explain this association of lesions in the lower colon and in the urinary bladder. The fact that the terminal portion of the colon receives its parasympathetic supply for S-2, 3, and 4 (that is, the pelvic parasympathetic system) suggests that congenital megacolon is a malformation in the pelvic parasympathetic system so that the terminal ganglion cells fail to develop. This would account for the constant location of the lesion in the distal colon. In those patients with the major portion of the colon or all of the colon aganglionic, one would have to postulate a lesion of the terminal portion of the vagal system.

The pelvic parasympathetic system also supplies the detrusor of the urinary bladder, and this fact seems important in the understanding of the patient with megalo-bladder. It would seem reasonable to consider that if one has a congenital lesion of the pelvic parasympathetic system, that this might involve bladder function as well as function of the terminal portion of the colon. Should this be true, it would be logical to postulate three groups of patients: one, patients with a lesion in the colon and an intact urinary bladder; two, patients with a defect in the terminal colon and in the bladder; and third, patients with an intact colon and a defect in bladder function alone. This last group of patients might include some of the patients with idiopathic megalo-ureters.

A group of 35 patients with megalo-ureters has come under my care. Cystometrograms have been utilized in classifying these patients. By our method, a normal cystometrogram begins with an initial zero pressure and gradually rises to 10 or $12 \mathrm{~cm}$. of water pressure before detrusor contractions begin and the bladder empties. In contrast to this, the patients with megalo-bladder have two or three times the capacity of a normal child of comparable age and size, and in addition, the pressure rises to as much as 25 or $35 \mathrm{~cm}$. of water pressure. Detrusor contractions may be absent, or during filling frequent low amplitude contractions may occur, but none during the terminal period of filling. By history these patients void only once or 
twice a day and tend to carry large residuals. I have performed ureteral peristalsis studies on these patients, and they have a tracing of active peristalsis that is perhaps somewhat different from normal tracings in that the rate of contraction may be a little slower than that in normal subjects. Ureteral contractions in a normal child occur at 7 to 10 -second intervals and the maximum pressure attained by the ureteral contraction varies from 35 to $45 \mathrm{~cm}$. of water. It is obvious that with intravesical pressure almost equalling the pressure generated by a peristaltic wave, the ureter will dilate. It was postulated that these children had defective bladders and that the dilatation of the upper urinary tract was secondary to a malfunctioning bladder. To test this hypothesis, the children were put either on urethral drainage or on suprapubic drainage, and, much to our satisfaction, there was a general improvement in the patients' conditions and in addition, a gratifying decrease in the size of the ureters and hydronephrosis. Prolonged catheter drainage has many complications and we have resorted to a different form of treatment in the last few years. In order to reduce the resistance to voiding, extensive resections of the bladder neck were performed, and by instructing the children to void frequently and to make a great effort to empty the bladder completely, gratifying results have been achieved. The rationale of this is to have these patients use the bladder where the range of pressure is near normal. An equally important part of the $\overrightarrow{\vec{F}}$ management of these children is to combat urinary $\stackrel{\vec{P}}{\rightarrow}$ infection. The parents and the local physicians are informed that these children must be considered in $\frac{\bar{F}}{\bar{N}}$ the same category as patients with rheumatic heart $\frac{\rho}{\sigma}$ disease. They must be carefully observed for a $\stackrel{\Phi}{\varrho}$ number of years. They should see their physician $\%$ at least once a month for residual determinations $\overrightarrow{0}$ and urine analysis. A wide variety of antibiotics is used to control the infection. It is most gratifying $\vec{\omega}$ to see how much can be accomplished with these children providing the child, the family and the local physician are willing to persevere (Fig. 3). The precise nature of the bladder dysfunction is difficult to ascertain. An opportunity presented $\vec{t}$ itself to study eight bladders removed from patients who had succumbed to the disease. Fortunately, 음 the radiographs and clinical records were available for study. The specimens removed at necropsy $T$ included the kidney, ureters, bladder and urethra. There was no evidence of mechanical obstruction in the lower urinary tract. The entire bladder was 2 cut into blocks and imbedded. It is important to $\overrightarrow{0}$ emphasize that the whole organ was thus imbedde



Fig. 3A.-Cystogram of patient with megalo-bladder and megaloureters due to an aparasympathetic bladder. Note bilateral reflux. Residual $150 \mathrm{ml}$.



Fig. 3B.-Cystogram on same patient three months after resection of the bladder neck. Residual $15 \mathrm{ml}$. This patient had adhered to a programme of voiding five times a day and once during the night. 
and histological sections were studied from each paraffin block. Therefore, the whole bladder was studied. We took a similar number of normal bladders and studied them in the same manner in order to determine the number and distribution of ganglion cells. It was found that in the normal specimen the concentration of ganglion cells was greatest in the peri-ureteral area on each side and that the concentration diminished rapidly in all directions from the ureteral orifice (Swenson, MacMahon, Jaques and Campbell, 1952).

In studying the specimens from patients with megalo-ureters and megalo-bladder a striking diminution in the number of ganglion cells was discovered. In some of the specimens the ganglion cells were extremely difficult to find. This lends support to our conception that these patients have a parasympathetic defect in the urinary bladder, and because of abnormal innervation they have enlarged bladder, high intravesical pressure, difficulty in emptying, and carry large residuals. A second group of patients with megalo-ureters was encountered which does not fit into this category. This group of patients has an essentially normal bladder, but upon study of ureteral function peristalsis is found to be absent. In one patient there was a rhythmic activity in the ureter, but actually the tracing was the reverse of that seen in normal patients so that the ureters seem to be intermittently dilated rather than contracted (Fig. 4). The cause of this dysfunction is obscure. Infection may play a role, but it is not the major factor, for a number of patients with infection have been demonstrated to have normal peristalsis. A congenital malformation is probably the underlying pathology. This

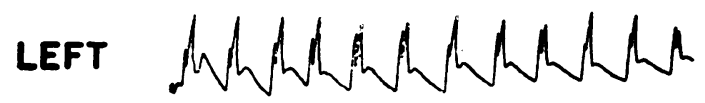

RIGHT

\footnotetext{
RIGHT

Fig. 4.-Ureteral peristalsis tracings from a patient with a normal bladder and right megalo-ureter. Note normal peristalsis in left ureter. On the right side no peristalsis could be detected during two long periods of observation.
}

is difficult to establish histologically for there are few ganglion cells in a normal ureter so that study of these neural elements has not proved helpful. Massive chronic dilatation probably plays a role. However, normal peristalsis has been demonstrated in ureters many times normal in size.

One practical point should be stressed and that is in the group with aparasympathetic bladders; nothing is gained by operation on the lower end of the ureter; in fact, irreparable damage may be done. I have seen two patients who, after bilateral resection of the lower ends of the ureter and reimplantation, were unable to void. These patients probably had a congenital defect in the pelvic parasympathetic system and the operative procedure destroyed what remaining innervation they had.

This group of patients has been the most difficult to treat. They have done poorly as a group and all that one can do is control the infection and, as their retention of nitrogenous products mounts, one can only resort to nephrostomy.

After careful study our group of 35 patients fall into the following groups: 17 had aparasympathetic bladder and four of these had Hirschsprung's disease as well; nine had absent ureteral peristalsis; four had cord bladder; three had ureterocoeles; and two had agenesis of the abdominal wall.

\section{Summary}

Hirschsprung's disease is due to a congenital malformation in the pelvic parasympathetic system. The absence of ganglion cells in the distal colon accounts for the absence of peristalsis in this segment of the intestinal tract. There is one group of patients with megalo-ureters and megalo-bladder in which the pathology is a defect in the parasympathetic innervation in the bladder. Ureteral peristalsis is essentially normal in this group of patients with megalo-ureters and megalo-bladder. There is a second group of patients with megalo-ureters, either unilateral or bilateral, in which the bladder is normal and peristalsis is absent or highly abnormal.

\section{REFERENCES}

Bodian, M., Stephens, F. D. and Ward, B. C. H. (1950). Lancet, 1, 19.

Dalla Valle, A. (1920). Pediatria, Napoli, 28, 740.

Hiatt, R. B. (1951). Ann. Surg., 133, 321 .

Robertson, H. E. and Kernohan, J. W. (1938). Proc. Mayo Clin., 13, 123.

State, D. (1952). Surg. Gynec. Obstet., 95, 201.

Swenson, O. and Bill, A. H., Jr. (1948). Surgery, 24, 212.

MacMahon, H. E., Jaques, W. E. and Campbell, J. S. (1952). New Engl. J. Med., 246, 41.

Rheinlander, H. F. and Diamond, I. (1949). Ibid., 241, 551.

Tiffin, M. E., Chandler, L. R. and Faber, H. K. (1940). Amer. J. Dis. Child., 59, 1071 .

Zuelzer, W. W. and Wilson, J. L. (1948). Ibid., 75, 40. 